\title{
Micromechanics of diffuse axonal injury: influence of axonal orientation and anisotropy
}

\author{
R. J. H. Cloots - J. A. W. van Dommelen - T. Nyberg • \\ S. Kleiven - M. G. D. Geers
}

Received: 28 February 2010 / Accepted: 1 July 2010 / Published online: 16 July 2010

(C) The Author(s) 2010. This article is published with open access at Springerlink.com

\begin{abstract}
Multiple length scales are involved in the development of traumatic brain injury, where the global mechanics of the head level are responsible for local physiological impairment of brain cells. In this study, a relation between the mechanical state at the tissue level and the cellular level is established. A model has been developed that is based on pathological observations of local axonal injury. The model contains axons surrounding an obstacle (e.g., a blood vessel or a brain soma). The axons, which are described by an anisotropic fiber-reinforced material model, have several physically different orientations. The results of the simulations reveal axonal strains being higher than the applied maximum principal tissue strain. For anisotropic brain tissue with a relatively stiff inclusion, the relative logarithmic strain increase is above $60 \%$. Furthermore, it is concluded that individual axons oriented away from the main axonal direction at a specific site can be subjected to even higher axonal strains in a stress-driven process, e.g., invoked by inertial forces in the brain. These axons can have a logarithmic strain of about 2.5 times the maximum logarithmic strain of the axons in the main axonal direction over the complete range of loading directions. The results indicate that cellular level heterogeneities have an important influence on the axonal strain, leading to an orientation and location-dependent sensitivity of the tissue to mechanical loads. Therefore, these effects should be
\end{abstract}

R. J. H. Cloots · J. A. W. van Dommelen $(\varangle) \cdot$ M. G. D. Geers Materials Technology Institute,

Eindhoven University of Technology,

P.O. Box 513, 5600 MB Eindhoven, The Netherlands

e-mail: j.a.w.v.dommelen@tue.nl

T. Nyberg $\cdot$ S. Kleiven

Division of Neuronic Engineering,

School of Technology and Health, Royal Institute of Technology,

14152 Huddinge, Sweden accounted for in injury assessments relying on finite element head models.

Keywords Traumatic brain injury (TBI) .

Diffuse axonal injury (DAI) - Brain tissue .

Finite element model $\cdot$ Cellular mechanics $\cdot$ Axons $\cdot$ Neurons

\section{Introduction}

Traumatic brain injury (TBI) can be caused by accidents, for instance in road traffic or sports, leading to serious health issues or even death. The incidence rate and mortality rate in Europe are estimated to be 235 and 15.4 per 100,000 of the population per year, respectively (Tagliaferri et al. 2006). One of the most frequently occurring types of TBI is diffuse axonal injury (DAI), which is primarily involved with dynamic non-contact loads, although it is believed to occur in closed head impacts as well and is associated with widespread injury in the brain (Gentleman et al. 1995; Smith et al. 2003).

The most commonly used brain injury criterion, which relates brain injury to mechanical loading, in the automotive industry is the head injury criterion (HIC) (Versace 1971). However, the application of HIC is limited, since it is based on experimental data of head accelerations, in which only anterior-posterior contact loading has been applied to human cadavers, not accounting for head angular accelerations or brain tissue strains and stresses. Therefore, finite element (FE) head modeling provides a more sophisticated method for assessing the likelihood of brain injury as a result of a mechanical load (Brands et al. 2002; Raul et al. 2006; Al-Bsharat et al. 1999; Takhounts et al. 2003; Kleiven 2006). These models simulate the consequences of mechanical loads on the head by predicting the stress and strain inside the brain. 


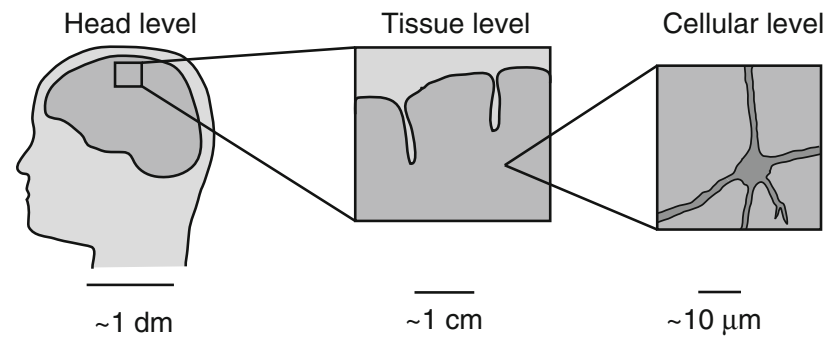

Fig. 1 The length scales involved with TBI ranging from decimeters for the head level to micrometers at the cellular level

However, at present, there is no direct link between mechanical load of the tissue and cellular injury.

To investigate the mechanophysical mechanisms of TBI, different length scales of the brain are distinguished (see Fig. 1). At the head level, the mechanical load is applied to and transmitted through the brain. In order to predict a true tissue stress and strain, the geometrical details of the brain are important because of the induced concentrations of stress and strain in the tissue (Bradshaw et al. 2001; Cloots et al. 2008; Lauret et al. 2009; Ho and Kleiven 2009). Even more, these tissue level strains will lead to a mechanical loading of brain cells, possibly leading to physiological damage. Therefore, it is assumed that investigating the mechanical phenomena at the cellular level might provide information about the fundamental triggers of DAI. At this level, the individual brain cells and their constituting elements can be distinguished. Neurons and glial cells consist of a soma (i.e., a cell body) and processes (i.e., axons and dendrites), which extend from the soma (Marieb 1998; Nolte 2002). Somal sizes are about $5 \mu \mathrm{m}$ in diameter for the glial cells and often less then $10 \mu \mathrm{m}$ for non-pyramidal neurons and up to $20 \mu \mathrm{m}$ for other cortical and hippocampal neurons (Rajkowska and Goldman-Rakic 1995; Rajkowska et al. 1998; Pierri et al. 2001; Cotter et al. 2002; Highley et al. 2003; Hutsler 2003). Most of the somata of the neurons are found in the cortex from which the axons extend into other parts of the brain. Axons have a uniform diameter and can be many centimeters long, whereas dendrites taper away from the soma and rarely exceed $500 \mu \mathrm{m}$ in length (Alberts et al. 1994). The majority of the brain tissue volume consists of axons. Even in the cerebral cortex, which is relatively rich in somata and blood vessels in comparison with other parts of the brain, about $5-10 \%$ consists of glial processes, $60-70 \%$ of neuronal processes (including their boutons and spines), 10-20\% of somata and blood vessels, and the remaining part is the extracellular space (Fenstermacher et al. 1970; Braitenberg and Schüz 1998; Ventura and Harris 1999; Chklovskii et al. 2002).

Povlishock (1993) found that DAI is not associated with direct mechanical tearing of axons in the white matter, but with the discrete focal impairment of axoplasmic transport leading to local axonal swelling and lobulation, which were all found at locations where the axon changed its anatomical course (e.g., near a blood vessel or a soma). Furthermore, Povlishock observed that damaged axons could be found intermingled with intact axons. In the current study, it is therefore assumed that these locally occurring injuries are caused by locally increased strains, since the brain is loaded mechanically prior to injury. Furthermore, it is assumed that axonal orientation and the presence of inclusions (e.g., a blood vessel or a soma) in the pathway of the axons are the main triggers of locally increased strains.

Computational mechanical modeling of the brain at the cellular level has been done to some extent (Arbogast and Margulies 1999; Khoshgoftar et al. 2007; Abolfathi et al. 2009; Karami et al. 2009). These studies were mainly focused on the relation between the tissue-level mechanical behavior and the cellular-level structures. This study, however, focuses on injury sensitivity as a function of the micromechanical characteristics. The aim is to assess the relation between tissue level mechanical loading and cellular level injury. Since local axonal damage has been observed for DAI, it is hypothesized that locally a higher strain occurs due to geometric or mechanical heterogeneities, constituting a precursor of local damage.

\section{Methods}

In this study, a plane strain FE model that relates tissuelevel mechanical loads to cellular-level brain injury has been developed. In the simulations, mechanical loads representing tissue-level deformations have been applied on a model with a length scale that is typical for individual cells. Based on the pathological findings mentioned in the Introduction, the model of interest contains typically one inclusion surrounded by axons. In order to determine which factors are critical for axonal strain concentrations, the axonal orientations and the mechanical properties are varied.

\subsection{Geometry}

The geometry of the model is based on the anatomical and pathological observations for axonal injury. The geometry contains a cylindrical inclusion (e.g., a blood vessel or a soma) with a diameter of $8 \mu \mathrm{m}$. It is assumed that the remaining part of the tissue consists of axons only. The interface between the inclusion and the surrounding tissue is assumed to be fully tied. The tissue surrounding the inclusion is modeled as a regular continuum where the behavior in each material point corresponds to the response of a mixture of axons. The spatial distribution of axonal orientations is either oriented or unoriented (i.e., random). For the oriented models, the axons are oriented fully uniaxially in every material 
Fig. 2 a Discretization and b-d axonal orientations of the model. The maximum diversion angle $\varphi$ is $\mathbf{b} 30^{\circ}, \mathbf{c} 45^{\circ}$, and $\mathbf{d}$ $60^{\circ}$. The outer dimensions are $50 \mu \mathrm{m}$ by $50 \mu \mathrm{m}$

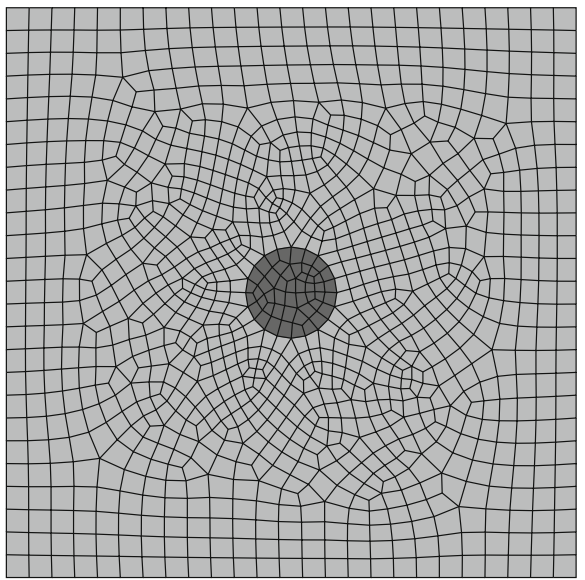

a

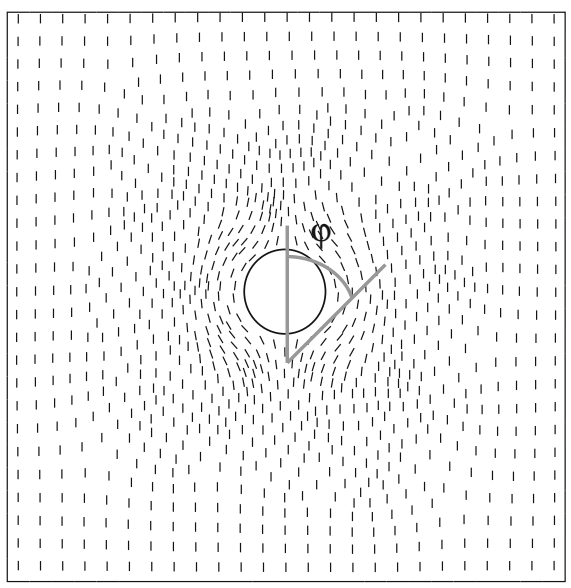

C

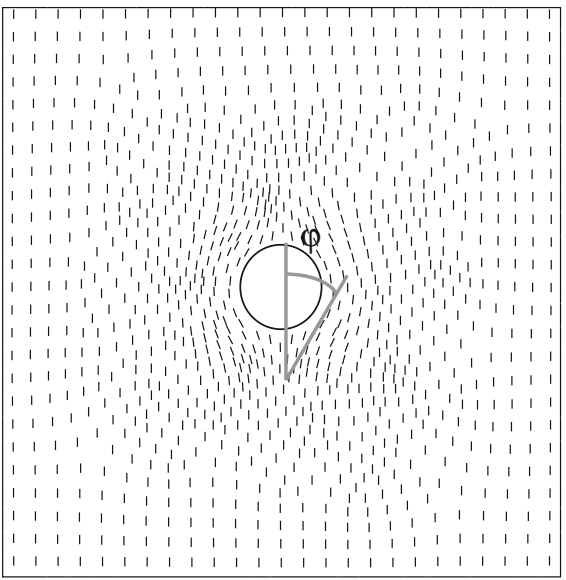

b

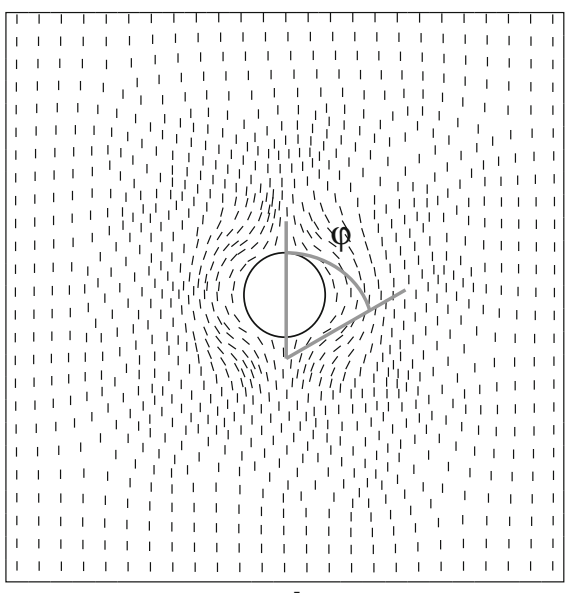

d point. However, the axonal orientation in different material points differs as shown in Fig. 2. The axons are diverting from the inclusion, and the amount of diversion is defined as the maximum angle $\varphi$ between the local axon orientation and the main axonal direction in the far field. To investigate the influence of the diversion angle, it is chosen to model configurations with $\varphi=30^{\circ}, 45^{\circ}$, and $60^{\circ}$. Also, analytical computations of the fiber-reinforced material model, which will be explained later, are used, representing a homogeneous geometry (i.e., without an inclusion) with oriented fibers. This situation is referred to as $\varphi=0^{\circ}$. To study the effect of the main axonal orientation, the principal loading direction is varied with respect the main axonal orientation, as will be detailed further on.

\subsection{Boundary conditions}

Since the part of the brain that is modeled here is in reality surrounded by adjacent brain tissue, periodic boundary conditions are adopted to include that influence (Kouznetsova et al. 2001):

$$
\begin{aligned}
& \left.\vec{u}\right|_{\Gamma_{34}}-\left.\vec{u}\right|_{\Gamma_{12}}=\left.\vec{u}\right|_{c_{4}}-\left.\vec{u}\right|_{c_{1}} \\
& \left.\vec{u}\right|_{\Gamma_{23}}-\left.\vec{u}\right|_{\Gamma_{14}}=\left.\vec{u}\right|_{c_{2}}-\left.\vec{u}\right|_{c_{1}}
\end{aligned}
$$

where $c_{i}$ are the corner nodes, $\Gamma_{i j}$ represent the boundaries of the model as shown in Fig. 3 and $\vec{u}$ represents the displacement vector. An isochoric overall deformation is applied, in which the principal strain direction is varied. This direction is indicated by the angle $\theta$ with respect to the global 1-direction of the model that corresponds with the main axonal direction. The deformation gradient tensor prescribed is defined as

$\overline{\boldsymbol{F}}=\bar{\lambda} \vec{m}_{1} \vec{m}_{1}+\frac{1}{\bar{\lambda}} \vec{m}_{2} \vec{m}_{2}+\vec{m}_{3} \vec{m}_{3}$

in which $\bar{\lambda}=1.01$ is the globally applied stretch ratio and the vectors $\vec{m}_{i}$ (for $i=1,2,3$ ) can be expressed in terms of the Cartesian vectors $\vec{e}_{j}$ (for $j=1,2,3$ ) and the angle $\theta$. The deformation gradient tensor is imposed on the unit cell in a standard manner through the displacements of the 

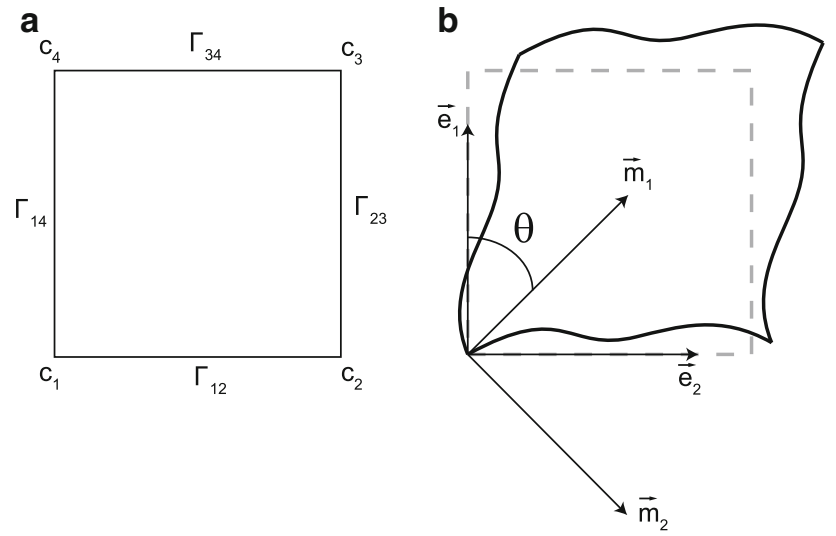

Fig. 3 a Labeling of the corner nodes and the boundaries. b Schematic representation of the deformation defined by the loading angle $\theta$ and the periodic boundary conditions

corner nodes $\mathrm{c}_{1}, \mathrm{c}_{2}$, and $\mathrm{c}_{4}$. This way, only the average deformation of the model is prescribed, whereas the exact local deformations follow from the equilibrium conditions and the heterogeneity of the model.

\subsection{Mechanical properties}

The mechanical behavior of brain tissue is not homogeneous throughout the brain. One marked aspect of the mechanical properties that shows interregional variation is the degree of anisotropy. Areas in which the axons are more aligned have a higher fractional anisotropy (FA), which is a measure to quantify the morphological anisotropy (Snook et al. 2005), as well as a higher mechanical anisotropy (Prange et al. 2000; Prange and Margulies 2002; Hrapko et al. 2008). Inside each axon, fibers are aligned in the direction of the axon itself (Alberts et al. 1994). These fibers are the neurofilaments, and they provide the axon its mechanical strength. The mechanical response of the brain tissue with a fibrous microstructure is described with the following anisotropic strain energy potential (Simulia, Providence, RI 2008):

$$
\begin{gathered}
W=G\left(\tilde{I}_{1}-3\right)+K\left(\frac{J^{2}-1}{4}-\frac{1}{2} \ln J\right) \\
+\frac{k_{1}}{2 k_{2}} \sum_{\alpha=1}^{N}\left(e^{k_{2}\left\langle\tilde{E}_{\alpha}\right\rangle^{2}}-1\right)
\end{gathered}
$$

where the third term on the right hand side is the HolzapfelGasser-Ogden form (Gasser et al. 2006) with

$\tilde{E}_{\alpha}=\kappa\left(\tilde{I}_{1}-3\right)+(1-3 \kappa)\left(\tilde{I}_{4 \alpha}-1\right)$

In Eq. (4), $W$ is the strain energy per unit of reference volume, $K$ is the bulk modulus, $G$ is the shear modulus $N$ is the number of fiber families, $\tilde{I}_{1}$ is the first invariant of the isochoric Cauchy-Green deformation tensor, $J=\operatorname{det}(\boldsymbol{F})$ is the volume ratio, and $k_{1}, k_{2}$, and $\kappa$ are material parameters for describing the behavior of the fibers. Furthermore, $\tilde{I}_{4 \alpha}=\tilde{\boldsymbol{C}}: \vec{n}_{0 \alpha} \vec{n}_{0 \alpha}$ where $\tilde{\boldsymbol{C}}=J^{-\frac{2}{3}} \boldsymbol{C}$ is the isochoric part of the right Cauchy-Green deformation tensor $\boldsymbol{C}$ and $\vec{n}_{0}$ is the fiber direction vector in the reference configuration with unit length. The material parameter $\kappa$ describes the dispersion of the fiber orientations around the preferred fiber direction $\vec{n}_{0 \alpha}$. The two limits of $\kappa$ are 0 for fully aligned fibers (i.e., full transverse anisotropy) and $\frac{1}{3}$ for randomly orientated fibers (i.e., isotropy). The Macaulay brackets $\langle\cdot\rangle$ impose $\left\langle\tilde{E}_{\alpha}\right\rangle$ to become 0 if $\tilde{E}_{\alpha}$ is negative, and therefore, the fibers contribute only in tension and not in compression. The Cauchy stress tensor is then expressed as

$\sigma=\sigma^{h}+\sigma^{d}$

The hydrostatic part is given by

$\boldsymbol{\sigma}^{h}=\frac{K}{2} \frac{J^{2}-1}{J} \boldsymbol{I}$

and the deviatoric part is

$$
\begin{array}{r}
\sigma^{d}=\frac{1}{J}\left(G \tilde{\boldsymbol{B}}^{d}+2 k_{1} \sum_{\alpha=1}^{N} e^{k_{2}\left\langle\tilde{E}_{\alpha}\right\rangle^{2}}\left\langle\tilde{E}_{\alpha}\right\rangle\right. \\
\left.\left(\kappa \tilde{\boldsymbol{B}}^{d}+\tilde{I}_{4 \alpha}(1-3 \kappa)\left(\vec{n}_{\alpha} \vec{n}_{\alpha}\right)^{d}\right)\right)
\end{array}
$$

where $\boldsymbol{I}$ is the unit tensor, and $\tilde{\boldsymbol{B}}^{d}$ is the deviatoric part of the isochoric Finger tensor $\tilde{\boldsymbol{B}}=J^{-\frac{2}{3}} \boldsymbol{B}$.

The material properties of the part consisting of axons only and the corresponding geometrical conditions are summarized in Table 1 for three different configurations. The material properties of the fiber-reinforced oriented axons are based on the study performed by Ning et al. (2006). Also, two other configurations are used: fiber-reinforced unoriented axons and oriented axons without fiber reinforcement. The fiberreinforced oriented configuration results in anisotropic tissue behavior, whereas the other two configurations result in

\begin{tabular}{|c|c|c|c|c|c|}
\hline \multirow[t]{2}{*}{ Configuration } & \multirow[t]{2}{*}{$G(\mathrm{~Pa})$} & \multirow{2}{*}{\multicolumn{2}{|c|}{$k_{1}(\mathrm{~Pa}) \kappa$}} & \multicolumn{2}{|c|}{$\varphi$} \\
\hline & & & & $\begin{array}{l}\text { Without } \\
\text { inclusion }\end{array}$ & $\begin{array}{l}\text { With } \\
\text { inclusion }\end{array}$ \\
\hline \multicolumn{6}{|c|}{ Fiber-reinforced tissue with } \\
\hline Oriented axons & 12.7 & 121.2 & 0 & $0^{\circ}$ & $30^{\circ} / 45^{\circ} / 60^{\circ}$ \\
\hline Unoriented axons & 12.7 & 121.2 & $\frac{1}{3}$ & N/A & N/A \\
\hline $\begin{array}{l}\text { Tissue without fiber } \\
\text { reinforcement, } \\
\text { but with oriented axons }\end{array}$ & 12.7 & 0 & 0 & $0^{\circ}$ & $30^{\circ} / 45^{\circ} / 60^{\circ}$ \\
\hline
\end{tabular}
isotropic tissue behavior. For the fiber-reinforced unoriented

Table 1 Brain tissue material properties and maximum fiber diversion angle $\varphi$ for three configurations 
axons, isotropy is caused by the random distribution of axons, which can be found closer to or within the cerebral cortex. For the oriented axons without fiber reinforcement, however, the fiber contribution to the stiffness is ignored. Note that this reduces Eq. 8 to a Neo-Hookean constitutive equation. The main reason to include the latter isotropic configuration is to investigate the separate effects of anisotropic stiffness and oriented axonal stretching. The material model is further simplified by using one fiber family (i.e., $N=1$ ) and therefore, the axons are locally fully aligned. However, the fiber orientation can be spatially heterogeneous, as described previously. Furthermore, fiber contribution to the stiffness is assumed to be linear, and therefore the non-linear parameter $k_{2} \rightarrow 0$ is used. With these assumptions, Eq. (4) can be rewritten as

$W=G\left(\tilde{I}_{1}-3\right)+K\left(\frac{J^{2}-1}{4}-\frac{1}{2} \ln J\right)+\frac{k_{1}}{2}\left\langle\tilde{E}_{1}\right\rangle^{2}$

The bulk modulus $K$ is assumed to be constant over the whole volume of the tissue and is reported to range from $2.1 \mathrm{GPa}$ (McElhany et al. 1976) to $2.5 \mathrm{GPa}$, based on the velocity of the dilatational waves in brain tissue of approximately $1550 \mathrm{~ms}^{-1}$ measured in ultrasonic experiments (Goldman and Hueter 1956; Etoh et al. 1994; Lin et al. 1997). In this study, a bulk modulus of $2.5 \mathrm{GPa}$ is chosen.

The inclusion is assumed to be isotropic, and its material properties are based on the surrounding tissue. In case the inclusion represents a cross-section of a soma, which is assumed to consist of similar material as the axons, the material properties are the same as the fiber-reinforced unoriented configuration for the brain tissue (see Table 1). For other cases (e.g., blood vessels), the material properties of the inclusion are varied in order to investigate the mechanical influence of the inclusion stiffness on the surrounding axons.

\subsection{Numerical approximation}

The model has been developed within Abaqus 6.8-2 (Simulia, Providence, RI 2008). A geometrically non-linear approach is adopted using a model that contains 1,076 quadrilateral bilinear elements with hourglass control and reduced integration because of the near incompressibility of brain tissue.

\subsection{Output quantities}

This study is especially focussed on the local consequences of a global mechanical load on brain tissue. As mentioned previously, DAI is found to be related to discrete focal impairment of axons (Povlishock 1993). Therefore, a relative measure of the axonal strain with respect to a global load measure is used. The axonal strain $\varepsilon$ is defined as the maximum logarithmic strain in the axonal direction, which is the material 1-direction for oriented axons and the maximum principal
Table 2 Summary of the output measures

\begin{tabular}{ll}
\hline Output measure & Description \\
\hline $\bar{\lambda}$ & Applied tissue stretch \\
$\bar{\varepsilon}=\ln (\bar{\lambda})$ & Applied tissue strain \\
$\varepsilon$ & Maximum axonal strain \\
$\tilde{\varepsilon}=\frac{\varepsilon}{\bar{\varepsilon}}$ & Maximum axonal strain relative \\
$\bar{\varepsilon}_{\sigma}=\frac{\varepsilon}{\left(\bar{\sigma}_{11}-\bar{\sigma}_{22}\right)}$ & to the applied tissue strain \\
$\bar{\varepsilon}_{\sigma, \text { ref }}$ & Maximum axonal strain relative \\
$\tilde{\varepsilon}_{\sigma}=\frac{\bar{\varepsilon}_{\sigma}}{\bar{\varepsilon}_{\sigma, \text { ref }}}$ & to the applied tissue stress \\
& Reference axonal strain relative \\
& to the applied tissue stress \\
\hline
\end{tabular}

strain direction for unoriented axons. By taking the maximum principal tissue strain $\bar{\varepsilon}=\ln (\bar{\lambda})$, which is imposed on the boundaries, as the global load measure, the relative strain becomes $\tilde{\varepsilon}=\frac{\varepsilon}{\bar{\varepsilon}}$.

During realistic loading conditions responsible for DAI, however, the tissue deformation is strongly driven by stress, because of the inertial forces acting on the brain. Therefore, also the maximum local strain with respect to the global normal stress difference in the tissue is considered. This measure is representative for a process driven by a uniaxial stress and is defined as $\bar{\varepsilon}_{\sigma}=\frac{\varepsilon}{\left(\bar{\sigma}_{11}-\bar{\sigma}_{22}\right)}$, where $\bar{\sigma}_{11}$ and $\bar{\sigma}_{22}$ are the homogenized stress components in the rotated coordinate system $\left\{\vec{m}_{1}, \vec{m}_{2}\right\}$, which are computed as in Kouznetsova et al. (2001). This normal stress difference corresponds to the maximum in-plane shear stress. Then, the axonal strain relative to the applied stress is normalized with this quantity for a reference situation, $\tilde{\varepsilon}_{\sigma}=\frac{\bar{\varepsilon}_{\sigma}}{\bar{\varepsilon}_{\sigma, \text { ref }}}$, where the reference situation is the homogeneous fiber-reinforced tissue with unoriented axons. The above-mentioned output measures are summarized in Table 2.

Besides relative or absolute axonal strains, an invariant stress measure will be used as well to investigate the influence of the global loads. For this, the equivalent stress $\sigma^{\mathrm{eq}}=\sqrt{\frac{3}{2} \sigma^{d}: \sigma^{d}}$ is used, in which $\sigma^{d}$ is the deviatoric part of the Cauchy stress tensor $\sigma$.

\section{Results}

This study is concerned with the global mechanical influences on axonal injury and therefore, the results, including the field plots, depict the tissue part that consists of axons, but not the inclusion. Figure 4 shows the results of the simulations with oriented fiber-reinforced axons and a maximum diversion angle of $45^{\circ}$ at three different loading angles. For both the equivalent stress and the axonal logarithmic strain, it is obvious that the values decrease for higher loading angles. Furthermore, at the same loading angles, maximum values are located similarly for the stress and strain fields. At a 

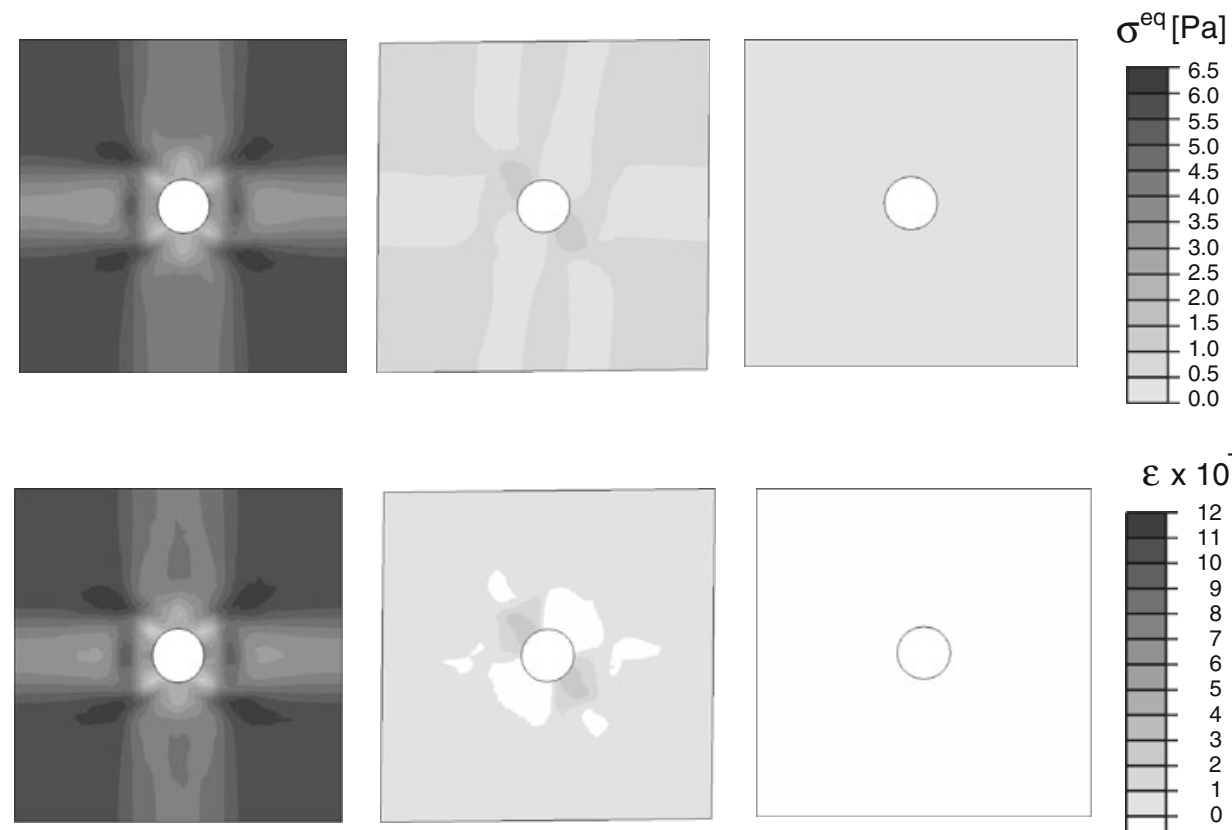

$\theta=0^{\circ}$

$45^{\circ}$

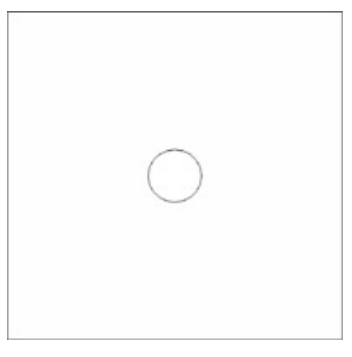

$\varepsilon \times 10^{-3}[-]$

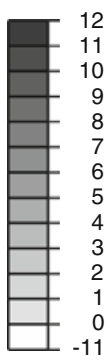

$90^{\circ}$

Fig. 4 Equivalent stress (upper row) and axonal logarithmic strain (lower row) fields of the fiber-reinforced model with a maximum diversion angle of $\varphi=45^{\circ}$, and principal loading directions at an angle of

$\theta=0^{\circ}, 45^{\circ}$, and $90^{\circ}$. Note that the stress and strain in the inclusion are not shown in these field plots

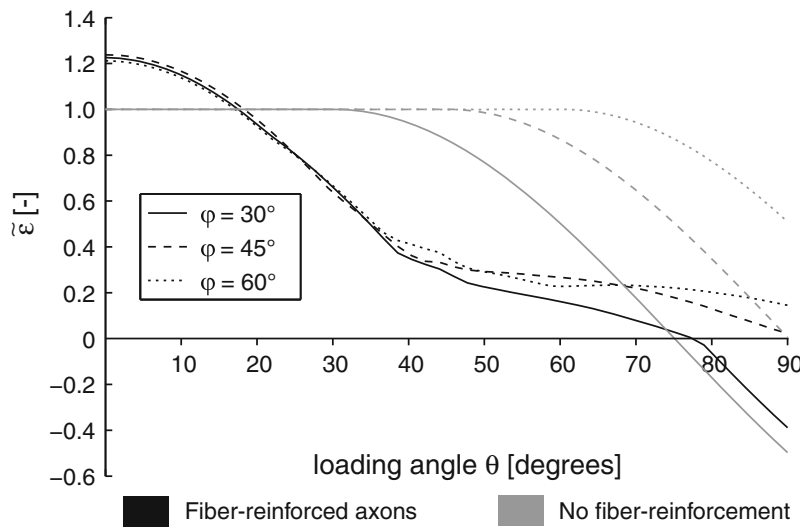

Fig. 5 The maximum relative local axonal strain as a function of the principal loading angle $\theta$ for the oriented fibers with maximum diversion angles of $\varphi=30^{\circ}, 45^{\circ}$, and $60^{\circ}$

loading angle of $\theta=0^{\circ}$, all axonal strain values are positive (i.e., all the axons are stretched), whereas all axonal strain values are negative (i.e., all the axons are compressed) at $\theta=90^{\circ}$.

In Fig. 5, the maximum relative axonal strains, which were defined previously, are plotted as a function of the loading angle for the configurations with the oriented fibers. Unoriented fiber configurations as well as the homogeneous ori-

ented fiber configuration are not shown, because the relative strain in these cases has a value of 1 over the entire range of loading directions. The highest maximum axonal strain relative to the globally applied strain is about 1.2 for the fiber-reinforced case. This value is obtained when the tissue is loaded in the main axonal direction. Large axonal strains are found for a small range of loading angles. In case of no fiber reinforcement, the local strain is never above the globally applied strain, and the maximum relative strain decreases depending on the maximum diversion angle. The maximum relative strain is more dependent on the loading direction for lower maximum diversion angles.

The maximum axonal strain relative to the applied stress normalized by a reference value for homogeneous tissue is depicted in Fig. 6 as a function of the loading angle for configurations with oriented fibers. Unoriented fiber configurations are not shown, because the normalized relative strain in these cases has a value of 1 over the entire range of loading directions. The results of the model without fiber-reinforced axon are the same as for the axonal strain relative to the tissue level strain. For loading angles above approximately $\theta=45^{\circ}$, this is also the case for results of simulations with the fiber-reinforced axons. At around a $45^{\circ}$ loading angle, the fiber-reinforced tissue reaches a normalized maximum axonal strain relative to the applied stress that is $2.4,2.5$, and 


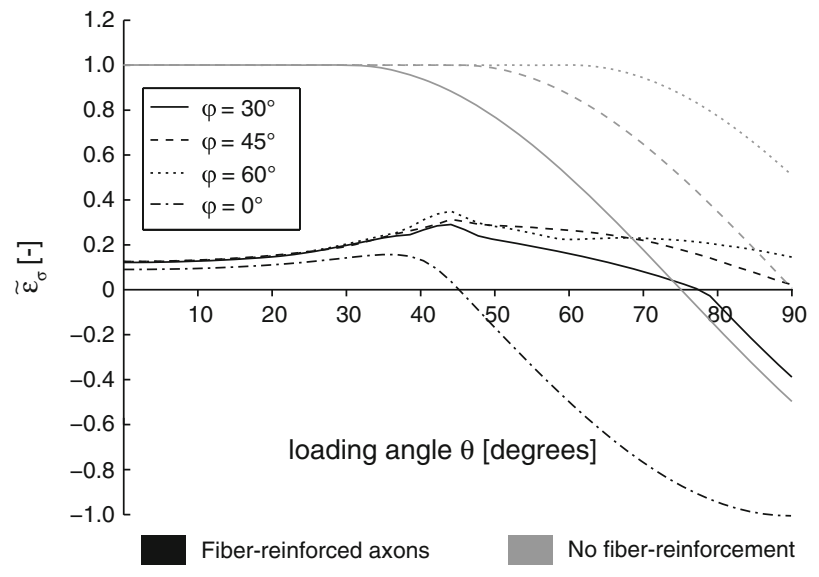

Fig. 6 The maximum local axonal strain relative to the applied stress as a function of the principal loading angle $\theta$ for the oriented fibers with maximum diversion angles of $\varphi=0^{\circ}, 30^{\circ}, 45^{\circ}$, and $60^{\circ}$

2.8 times the value obtained at the loading direction in the main axonal direction (i.e., $\theta=0^{\circ}$ ) for $\varphi=30^{\circ}, 45^{\circ}$, and $60^{\circ}$, respectively. For lower loading angles, the normalized maximum strains relative to the applied stress are lower than for the $45^{\circ}$ loading angle, because of the stiffening effect of the fibers that are oriented closely toward the loading angle. For higher loading angles, however, the normalized maximum strain relative to the applied stress decreases, because it is affected by the decrease in the axonal strain relative to the applied strain. The homogeneous oriented fiber configuration (i.e., $\varphi=0^{\circ}$ ) has a peak value at around $\theta=36^{\circ}$, which is 1.7 times the value obtained at $\theta=0^{\circ}$. For higher loading angles, the normalized maximum axonal strain relative to the applied stress drops to lower values more rapidly than the heterogeneous orientations (i.e., $\varphi=30^{\circ}, 45^{\circ}, 60^{\circ}$ ). Furthermore, it is observed that the peak values for the heterogeneous configurations are a factor of 1.8-2.2 with respect to the peak value reached for the homogeneous configuration. For the loading direction in the main axonal direction, the normalized maximum strain relative to the applied stress for the heterogeneous cases is increased by a factor 1.3-1.4 with respect to the homogeneous case.

In Fig. 7, the strain in the axonal direction is displayed for the simulations with $\varphi=45^{\circ}$ at a $0^{\circ}$ loading angle for two different values of the relative inclusion stiffnesses, which is defined as $\frac{G_{\text {inclusion }}}{G_{\text {tissue }}}=\frac{k_{1 \text {,inclusion }}}{k_{1, \text { tissue }}}$. For all cases, it can be noticed that a higher relative inclusion stiffness results in higher axonal strains. If only the subset of the three different configurations with a relative stiffness of 1 are compared to each other, it is observed that axonal alignment causes a heterogeneous strain field. Axonal alignment only leads to locally lower axonal strains, whereas alignment in combination with fiber reinforcement causes locally higher axonal strains. In case the relative stiffness is 10 , the axonal strain distribution is similar for both the fiber-reinforced unoriented axons

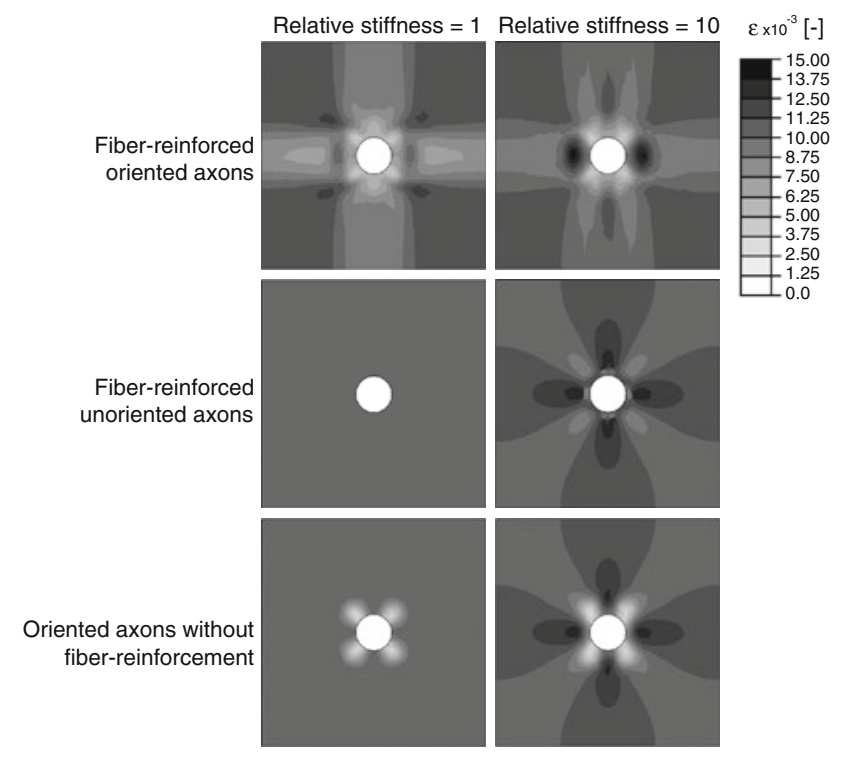

Fig. 7 Strain in the axonal direction for the simulations with a loading angle $\theta=0^{\circ}$ and different relative stiffnesses of the inclusion. For the oriented configurations, a maximum diversion angle of $45^{\circ}$ is used. Note that the strain in the inclusion is not shown in these field plots

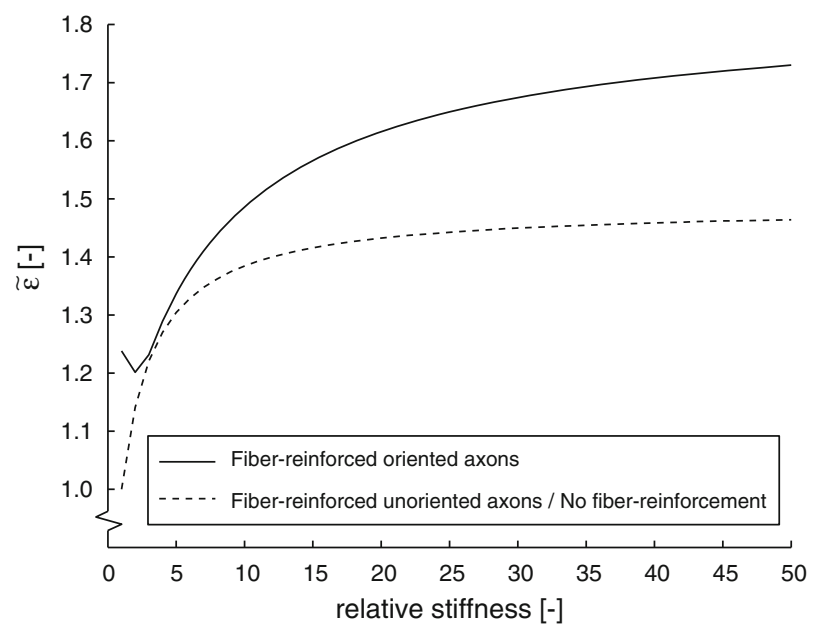

Fig. 8 Axonal strain relative to the applied strain as a function of the stiffness of the inclusion with respect to the tissue stiffness for the simulations with a maximum diversion angle of $\varphi=45^{\circ}$ at a loading angle of $\theta=0^{\circ}$

and the oriented axons without fiber reinforcement (i.e., the two situations with isotropic tissue behavior). Maximum values are located equatorial as well as polar to the inclusion. However, the locations with lower axonal strain in the latter configuration are similar to those observed for the situation with a relative stiffness of 1 . The configuration with fiberreinforced oriented axons and an inclusion stiffness 10 times the surrounding tissue results in maximum values that are located equatorial to the inclusion only.

In order to investigate the influence of the stiffness of the inclusion on the axonal strain more carefully, the maximum 
relative axonal strain is plotted against the relative inclusion stiffness in Fig. 8. For a high relative stiffness of the inclusion and fiber-reinforced oriented axons, the maximum axonal strain can exceed 1.7 times the global maximum principal tissue strain. This value is approximately 1.4 for the fiberreinforced unoriented axons as well as for the oriented axons without fiber reinforcement.

\section{Discussion}

From the results of all simulations, the most general observation is that the heterogeneities in the mechanical properties of brain tissue induce axonal strains higher than the applied tissue strain. The maximum local strains are all found near a stiff inclusion for axons aligned with the principal strain direction. The principal strain direction has an important influence on the results. For the fiber-reinforced oriented axons, the highest maximum axonal strains relative to the principal tissue strain are found at a $0^{\circ}$ loading angle, which is the main axonal direction. However, the highest normalized maximum axonal strain relative to the principal tissue stress occurs at a loading angle of approximately $45^{\circ}$ independently of the three different maximum diversion angles for the heterogeneous configurations. For the homogeneous case with fiber-reinforced oriented fibers, this occurs at about $36^{\circ}$. The reason the loading direction of the maximum axonal strain is shifted away from the main axonal direction is the stiffening effect of the fibers in the main axonal direction. Nevertheless, this effect is counteracted by the decrease in the axonal strain relative to the applied tissue strain for higher loading angles. The heterogeneous fiber-reinforced cases result in higher normalized maximum axonal strains relative to the applied stress than for the homogeneous case. For a loading direction in the main axonal direction, this factor is between 1.3 and 1.4 and is caused by the heterogeneous distribution of stiffnesses. For the peak values, the factor becomes 1.8-2.2. Here, also the fiber diversion angles have an influence on the normalized maximum axonal strain relative to the applied stress.

In this paper, it is assumed there is a relation between mechanical deformation and injury at an axonal level based on the observation by Povlishock (1993) that mechanically induced DAI results in local axonal injury positioned where the axon changes its anatomical course. The results of the simulations show strain concentrations under similar circumstances, which is an indication that such a relationship might exist. Also, in experimental studies it was shown that a mechanical load imposed on axons causes physiological or functional impairments (Bain and Meaney 2000; Bain et al. 2001; Galbraith et al. 1993).

For the development of a model that relates the mechanical aspects of TBI between the tissue level and the cellular level, several assumptions were made, such as for the geometry and the mechanical properties at the cellular level. Despite the complexity of the real structures, a relatively simple model geometry was adopted in order to systematically compare different situations within the limits set by the limited information on the mechanical properties at the cellular level. Since no complex loading conditions are considered, it is assumed that the material behavior can be modeled completely elastic. Furthermore, the applied strain levels are chosen to be small and are well below values commonly associated with DAI. However, under the current assumption that no large nonlinearities are present in the material behavior, the observed strain concentrations are almost independent of the strain level chosen. Relative quantities of predicted values are considered to be more important than absolute values making the conclusions drawn from this study partially independent of the chosen mechanical properties and loading conditions.

According to Lu et al. (2006), the storage modulus of glial cell processes and pyramidal cell processes amounts to onethird of the modulus of their respective somata. However, this is measured by indenting the processes in a direction perpendicular to the axis of the process. For the material model used in the current study, the inclusion has a lower stiffness compared to an axon in the axonal direction, but it has a higher stiffness compared to an axon in the direction perpendicular to the axonal direction. In the latter case, this factor of one-third is reached for a logarithmic strain of about 0.5 .

Since the model is plane strain, the inclusion has in fact a cylindrical shape, which could represent a blood vessel. However, the initially chosen material properties are based on the assumption that the inclusion is a soma, after which they were varied to investigate the effect of other types of inclusions (e.g., a blood vessel). A future study with threedimensional models should elucidate the effects of different inclusion geometries with a three-dimensional nature.

In the current study, the effects of a possible undulation of the axons have not been accounted for. Although the mechanical effect of the undulation could be implemented by the non-linear constant $k_{2}$ at the tissue level, this would lead to a violation of length scales in the current model. It has been concluded by Bain et al. (2003) that already at zero strain, parts of the axons are fully coupled to their surroundings, which causes them to deform in an affine manner with respect to their surroundings. Although the axonal strain relative to the applied tissue stress is expected to become less dependent of the loading direction as a result of the undulation for small strains, it is expected to have no influence for larger strains at which DAI could occur and the axons are not undulated anymore.

\section{Conclusion}

In this study, the relation between mechanical loading of brain tissue and axonal stretching is investigated, providing 
a bridge between different length scales involved in TBI. Axonal strains resulting from a deformation at the tissue level can become higher than the applied tissue strain. Values of over 1.7 for the relative axonal strain are found in the simulations with a stiff inclusion. The maximum values are reached when the principal loading direction is aligned with the main axonal direction. For the loading conditions that represent a stress-driven deformation, which is more representative for the mechanisms leading to TBI, axonal strains are shown to be influenced heavily by the fiber reinforcement of the axons. The peak normalized maximum axonal strain relative to the applied stress is reached when the angle between the loading direction and the main axonal direction is $36^{\circ}$ and about $45^{\circ}$ for the homogeneous and heterogeneous configurations, respectively. The peak normalized maximum axonal strain relative to the applied stress is between 1.8 and 2.2 times higher for the heterogeneous cases than for the homogeneous case.

In the results of the simulations, the location of the peak strain is found close to or within the axons that deviate from the main axonal direction. Axonal injury has also been observed in a pathological study at locations where axons are forced to deviate (Povlishock 1993). Factors that influence the increase of the axonal strain relative to the applied tissue strain or stress are found to be: (i) the inclusion stiffness with respect to the surrounding tissue, (ii) the axonal orientation, (iii) the fiber reinforcement (i.e., the effect of the neurofilaments), and (iv) the maximum diversion angle of the axons. Factor (iii) and especially factor (iv) have an important influence mainly on the axonal strain relative to the applied stress.

The heterogeneities at the cellular level cause increased axonal strains that might lead to local injury of an axon as a result of the tissue-level mechanical load, and as a consequence, a strong orientation-dependent sensitivity of axonal stretching to tissue level deformations is found. Therefore, FE head models should account for the effects of the mechanical heterogeneities at the cellular level and this orientationdependent sensitivity to predict more accurately DAI by means of anisotropic and microstructure-related injury criteria. Even though the current study indicates the importance of the cellular level with respect to TBI, more research is needed to properly quantify such injury criteria. Because it is not realistic to develop an FE head model with a resolution typical for the axonal length scale, a proper way to achieve this is the integration of a detailed micromechanical model in a macroscopic head model using a multiple scale approach, in which FE models representing different discrete length scales of the same material are coupled. It is expected that this will result in more realistic deformation modes and hence also more realistic cellular-level injury predictions. This will be the subject of future work. Depending on the outcome of that work, the effects of the heterogeneity and orientation of the axonal microstructure might be accounted for in anisotropic tissue-level injury criteria. In that case, ultimately, macroscopic head model simulations can account for the cellular-level effects by merely applying these injury criteria.

Acknowledgments This work has been supported by the Dutch Technology Foundation STW, applied science division of NWO and the Technology Program of the Ministry of Economic Affairs.

Open Access This article is distributed under the terms of the Creative Commons Attribution Noncommercial License which permits any noncommercial use, distribution, and reproduction in any medium, provided the original author(s) and source are credited.

\section{References}

Abolfathi N, Naik A, Chafi MS, Karami G, Ziejewski M (2009) A micromechanical procedure for modelling the anisotropic mechanical properties of brain white matter. Comput Method Biomech 12:249-262

Al-Bsharat AS, Hardy WN, Yang KH, Khalil TB, Tashman S, King AI (1999) Brain/skull relative displacement magnitude due to blunt head impact: new experimental data and model. In: Stapp car crash conference proceedings, vol 43. The Stapp Association.

Alberts B, Bray D, Lewis J, Raff M, Roberts K, Watson JD (1994) Molecular biology of the cell, 3rd edn. Garland Publishing, New York

Arbogast KB, Margulies SS (1999) A fiber-reinforced composite model of the viscoelastic behavior of the brainstem in shear. J Biomech $32: 865-870$

Bain AC, Meaney DF (2000) Tissue-level thresholds for axonal damage in an experimental model of central nervous system white matter injury. J Biomech Eng 122:615-622

Bain AC, Raghupathi R, Meaney DF (2001) Dynamic stretch correlates to both morphological abnormalities and electrophysiological impairment in a model of traumatical axonal injury. J Neurotrauma 18:499-511

Bain AC, Schreiber DI, Meaney DF (2003) Modeling of microstructural kinematics during simple elongation of central nervous system tissue. J Biomech Eng 125:798-804

Braitenberg V, Schüz A (1998) Cortex: statistics and geometry of neuronal connectivity, 2 nd edn. Springer, Berlin

Bradshaw DRS, Ivarsson J, Morfey CL, Viano DC (2001) Simulation of acute subdural hematoma and diffuse axonal injury in coronal head impact. J Biomech 34:85-94

Brands DWA, Bovendeerd PHM, Wismans JSHM (2002) On the potential importance of non-linear viscoelastic material modelling for numerical prediction of brain tissue response: test and application. In: Stapp car crash conference proceedings, vol 46. The Stapp Association

Chklovskii DB, Schikorski T, Stevens CF (2002) Wiring optimization in cortical circuits. Neuron 34:341-347

Cloots RJH, Gervaise HMT, van Dommelen JAW, Geers MGD (2008) Biomechanics of traumatic brain injury: influences of the morphologic heterogeneities of the cerebral cortex. Ann Biomed Eng 36:1203-1215

Cotter D, Mackay D, Chana G, Beasley C, Landau S, Everall IP (2002) Reduced neuronal size and glial cell density in area 9 of the dorsolateral prefrontal cortex in subjects with major depressive disorder. Cereb Cortex 12:386-394

Etoh A, Mitaku S, Yamamoto J, Okano K (1994) Ultrasonic absorption anomaly of brain tissue. Jpn J Appl Phys 33:2874-2879 
Fenstermacher JD, Li C, Levin VA (1970) Extracellular space of the cerebral cortex of normothermic and hypothermic cats. Exp Neurol 27:101-114

Galbraith JA, Thibault LE, Matteson DR (1993) Mechanical and electrical responses of the squid giant axon to simple elongation. J Biomech Eng 115:13-22

Gasser TC, Ogden RW, Holzapfel GA (2006) Hyperelastic modelling of arterial layers with distributed collagen fibre orientations. J Roy Soc Interface 3:15-35

Gentleman SM, Roberts GW, Gennarelli TA, Maxwell WL, Adams JH, Kerr S, Graham DI (1995) Axonal injury: a universal consequence of fatal closed head injury. Acta Neuropathol 89:537-543

Goldman DE, Hueter TF (1956) Tabular data of the velocity and absorption of high-frequency sound in mammalian tissue. J Acoust Soc Am 28:35-37

Highley JR, Walker MA, McDonald B, Crow TJ, Esiri MM (2003) Size of hippocampal pyramidal neurons in schizophrenia. Brit J Psychiatry 183:414-417

Ho J, Kleiven S (2009) Can sulci protect the brain from traumatic injury? J Biomech 42:2074-2080

Hrapko M, van Dommelen JAW, Peters GWM, Wismans JSHM (2008) The influence of test conditions on characterisation of the mechanical properties of brain tissue. J Biomech Eng 130:031003

Hutsler JJ (2003) The specialized structure of human language cortex: pyramidal cell size asymmetries within auditory and languageassociated regions of the temporal lobes. Brain Lang 86:226-242

Karami G, Grundman N, Abolfathi N, Naik A, Ziejewski M (2009) A micromechanical hyperelastic modeling of brain white matter under large deformation. J Mech Behav Biomed 2:243-254

Khoshgoftar M, Najarian S, Farmanzad F, Vahidi B, Ghomshe FT (2007) A biomechanical composite model to determine effective elastic moduli of the CNS gray matter. Am J Appl Sci 4:918-924

Kleiven S (2006) Evaluation of head injury criteria using a finite element model validated against experiments on localized brain motion, intracerebral acceleration, and intracranial pressure. Int J Crashworthiness 11:65-79

Kouznetsova VG, Brekelmans WAM, Baaijens FPT (2001) An approach to micro-macro modeling of heterogenous materials. Comp Mech 27:37-48

Lauret C, Hrapko M, van Dommelen JAW, Peters GWM, Wismans JSHM (2009) Optical characterization of acceleration-induced strain fields in inhomogeneous brain slices. Med Eng Phys 31:392399

Lin S, Shieh S, Grimm MJ (1997) Ultrasonic measurements of brain tissue properties. In: Proceedings of the 7 th injury prevention through biomechanics symposium, Centers for Disease Control, Wayne State University, pp 27-31

Lu Y, Franze K, Seifert G, Steinhäuser C, Kirchhoff F, Wolburg H, Guck J, Jammey P, Wei E-Q, Käs J, Reisenbach A (2006) Viscoelastic properties of individual glial cells and neurons in the
CNS. In: Proceedings of National Academy of Sciences, vol 103, pp 17759-17764. National Academy of Sciences

Marieb EN (1998) Human anatomy and physiology, 4th edn. Benjamin/ Cummings Science Publishing, Menlo Park

McElhany JH, Roberts VL, Hilyard JF (1976) Handbook of human tolerance, 2nd edn. Japan Automobile Research Institute Inc, Tokyo

Ning X, Zhu Q, Lanir Y, Margulies SS (2006) A transversely isotropic viscoelastic constitutive equation for brainstem undergoing finite deformation. J Biomech Eng 128:925-933

Nolte J (2002) The human brain: an introduction to its functional anatomy, 5th edn. Mosby, Inc., St. Louis, MO

Pierri JN, Volk CLE, Auh S, Sampson A, Lewis DA (2001) Decreased somal size of deep layer 3 pyramidal neurons in the prefrontal cortex of subjects with schizophrenia. Arch Gen Psychiatry 58:466473

Povlishock JT (1993) Pathobiology of traumatically induced axonal injury in animals and man. Ann Emerg Med 22:980-986

Prange MT, Margulies SS (2002) Regional, directional, and age-dependent properties of the brain undergoing large deformation. J Biomech Eng 124:244-252

Prange MT, Meaney DF, Margulies SS (2000) Defining brain mechanical properties: effects of region, direction, and species. In: Stapp car crash conference proceedings, vol 44. The Stapp Association

Rajkowska G, Goldman-Rakic PS (1995) Cytoarchitectonic definition of prefrontal areas in the normal human cortex: I. Remapping of areas 9 and 46 using quantitative criteria. Cereb Cortex 5:307-322

Rajkowska G, Selemon LD, Goldman-Rakic PS (1998) Neuronal and glial somal size in the prefrontal cortex. Arch Gen Psychiatry 55:215-224

Raul JS, Baumgartner D, Willinger R, Ludes B (2006) Finite element modelling of human head injuries caused by a fall. Int J Leg Med 120:212-218

Simulia (2008) Providence, R.I. Abaqus 6.8 manual

Smith DH, Meaney DF, Shull WH (2003) Diffuse axonal injury in head trauma. J Head Trauma Rehabil 18:307-316

Snook L, Paulson L, Roy D, Philips L, Beaulieu C (2005) Diffusion tensor imaging of neurodevelopment in children and young adults. Neuroimage 26:1164-1173

Tagliaferri F, Compagnone C, Korsic M, Servadei F, Kraus J (2006) A systematic review of brain injury epidemiology in Europe. Acta Neurochir 148:255-268

Takhounts EG, Eppinger RH, Campbell JQ, Tannous RE, Power ED, Shook LS (2003) On the development of the SIMon finite element head model. In: Stapp car crash conference proceedings, vol 47. The Stapp Association

Ventura R, Harris K (1999) Three-dimensional relationships between hippocampal synapses and astrocytes. J Neurosci 19:6897-6906

Versace J (1971) A review of the severity index. In: Stapp car crash conference proceedings, vol 15. The Stapp Association 